\title{
Christus in unsere heutigen Kontexte kommunizieren Christologische Zugänge zu einer Theologie der Kommunikation ${ }^{1}$
}

\author{
Ludwig Kaufmann, \\ einer Koryphäe \\ christopraktischer Kommunikation, \\ zum Gedenken
}

von Edmund Arens

\section{Der kommunizierte Jesus und der kommunizierende Christus}

Jesus wird auf vielfältige Weise in unsere heutigen Kontexte kommuniziert. Seine Person und sein Geist werden durch eine Vielzahl von Boten und Botschaften, Vermittlern und Medien in unsere sozio-politischen und kulturellen Kontexte hinein übermittelt. Seine Geschichte wird erzählt und sein Bild wird verbreitet durch die Imagination von Myriaden von Interpreten und Künstlern, Anhängern und Nachfolgenden. Christus in unsere gegenwärtige Lebenswelt zu kommunizieren, ist in einem eine hermeneutische, eine kritische wie eine konstruktiv-kreative Aufgabe. Darum verlangt sie einen Zugang zu den Quellen und deren Übersetzung in unsere Situation, historische wie systematische Kritik sowohl der Texte als auch unseres Tuns und eine Rekonstruktion bzw. Rekreation ihres ursprünglichen Interesses und ihrer Intention, ihres Impulses und ihrer Wirkung.

Im Neuen Testament wird die Kommunikation Jesu mit der Verkündigung in Verbindung gebracht. Verkündigung ist in der Tat Kommunikation des Evangeliums. Betrachten wir das Neue Testament, so erkennen wir, daß der Begriff „Evangelium“ von Beginn der christlichen Tradition an zweierlei bedeutet. Einerseits bezieht er sich auf die Geschichte Jesu, seine Person und Praxis sowie Gottes Handeln in und an ihm. Diese ganze Geschichte wird im „Evangelium Jesu Christi“ (Mk 1,1) erzählt. Andererseits ist Jesus nicht allein Inhalt bzw. Gegenstand des Evangeliums. Jesus Christus selbst erzählt seine Geschichte; durch das Evangelium kommuniziert er sein Wort. Das Evangelium von Jesus ist demnach zugleich die Selbstmitteilung Jesu Christi, in welcher der Logos durch den Geist redend und handelnd am Werk ist. Dieses schon in den synoptischen Evangelien implizierte Verständnis wird im Johannesevangelium explizit. Und es kommt nicht von ungefähr, daß "Communio et Progressio", wenn der Text auf die Kommunikation Christi, die "Geist und Leben" (CP 11) ist, zu sprechen kommt, auf Joh 6,63 Bezug nimmt, wo auf die Einsetzung der Eucharistie angespielt wird. Darauf wird eingegangen im Kontext der Selbstoffenbarung Jesu als Gottes Zeuge und Wort, als der Weg, die Wahrheit und das

Dr. Edmund Arens ist Privatdozent für Fundamentaltheologie an der Westfälischen-Wilhelms-Universität in Münster. Er lebt in Frankfurt. 
Leben. Von daher sollte jeder, der sich mit der Frage beschäftigt, wie Jesus in unsere heutigen Kontexte kommuniziert wird, zugleich den anderen Pol im Auge behalten, das heißt die Tatsache, daß Christus in unsere heutigen Kontexte hinein spricht.

Beide Dimensionen im Blick haltend, werde ich zunächst vier gegenwärtige Zugänge zur Christologie skizzieren, um das zu formulieren, was ich als deren verschiedene christologische Modelle der Kommunikation ansehe. Im Anschluß daran rekurriere ich auf ein Kommunikationsmodell, das ich aus der gegenwärtigen pragmatischen Theoriebildung in der Philosophie und den Sozialwissenschaften extrahiere. Darin sollen die diversen Dimensionen der Kommunikation zum Vorschein kommen. Drittens werde ich versuchen, einige Leitlinien für die Kommunikation Christi zu erarbeiten, die sich auf die Dimensionen der Kommunikation beziehen. Sie dienen dazu, die Bedingungen zu formulieren, die eine theologische Reflexion auf die Kommunikation Christi zu berücksichtigen hat, sowie die Ziele, die sie zu erreichen sucht. Schließlich wird die Kommunikation Christi auf die christliche Gemeinschaft bezogen. Ausgehend von der Einsicht, daß es sich bei der Kirche um eine bestimmte Kommunikationsgemeinschaft handelt, werden zwei Handlungen expliziert, die beide der Formulierung, der Bewahrung sowie der Weiterführung der Kommunikation Christi dienen.

\section{Zugänge zur Christologie}

Innerhalb der gegenwärtigen katholischen Theologie lassen sich m. E. vier prominente Zugänge zur Christologie unterscheiden. Sie beziehen sich auf verschiedene Aspekte des Jesus-Geschehens, nähern sich ihm und entfalten seine Bedeutung auf verschiedene Weise, geben unterschiedliche Antworten auf die Frage, wie Jesus Christus in unsere heutigen Kontexte zu kommunizieren sei. Die hier in aller Kürze vorzustellenden vier Ansätze nehmen ihren Ausgang entweder von der Anthropologie, von der Hermeneutik, der Ästhetik oder der Praxis. Sie sind von Karl Rahner, Edward Schillebeeckx, Hans Urs von Balthasar und von politischen bzw. Befreiungstheologen erarbeitet worden.

Rahners Christologie wurzelt in seiner Anthropologie; sie ist in der Tat eine theologische Entfaltung transzendentaler Anthropologie. Rahner hat seinen Ansatz im wesentlichen in seinen berühmten Lexikabeiträgen ${ }^{2}$ präsentiert und in seinem "Grundkurs des Glaubens ${ }^{\text {“3 }}$ zusammenfassend dargelegt. Sein Ansatz läßt sich zugleich als Explikation dessen auffassen, was Selbstmitteilung Gottes heißt. Rahner zufolge geschieht diese Selbstmitteilung auf zweifache Weise. Einerseits vollzieht sie sich immer schon existentiell durch die Gnade, die er als übernatürliches Existential begreift; andererseits teilt sich Gott geschichtlich auf einzigartige und unüberbietbare Weise in Jesus Christus mit. Nach Rahners Auffassung läßt sich Gottes Selbstmitteilung in Jesus Christus durch eine kombinierte philosophisch-anthropologische sowie existential-theologische Reflexion explizieren. Eine transzendentale Christologie erhellt die anthropologischen Voraussetzungen im Sinne der Erarbeitung der Bedingungen der Möglichkeit, die es Menschen erlauben, von Christus erreicht zu werden. Die transzendentale Christologie fragt von daher nach den apriorischen Möglichkeiten, die gegeben 
sein müssen, damit Menschen von der Botschaft und Person Jesu Christi angesprochen werden. Sie entfaltet das, wonach Menschen als Menschen immer schon fragen, was sie tun und erstreben, und sie arbeitet die Selbsttranszendenz als ein konstitutives Element menschlichen Seins, welches immer zugleich Werden beinhaltet, heraus. Rahner bezieht seinen transzendental-anthropologischen Zugang auf eine „suchende Christologie“" ${ }^{“ 4}$ in der Menschen, indem sie eine bestimmte Weise der Existenz führen, auf Christus ausgerichtet sind, ob sie dies nun wissen oder nicht. Solch eine suchende Christologie wird von Leuten vollzogen, die radikal und absolut ihre Nächsten lieben, die bereit sind, den Tod auf sich zunehmen und die für sich und für alle eine absolute, versöhnende Zukunft erhoffen.

Laut Rahner muß die christologische Lehre der Kirche auf diese menschlichen Fragen, Sehnsüchte und Handlungen Bezug nehmen in einer Weise, daß sie Christus als die Erfüllung des menschlichen Heilsverlangens präsentiert. Das Christentum behauptet Christus als Gottes kategorische Antwort auf die menschliche Selbsttranszendenz und das menschliche Heilsverlangen. In ihm hat Gott nicht allein etwas über sich selbst mitgeteilt, vielmehr hat er sich selbst mitgeteilt. Dies geschieht im wesentlichen in der Inkarnation, die für Rahner die fundamentale Selbstaussage und Selbstzusage Gottes darstellt. Die Inkarnation ist damit die geschichtliche Realisienung der Selbstmitteilung Gottes, in der der Geber zugleich die Gabe ist. Das durch die Inkarnation Gottes in der Wèlt verwirklichte Heil wird durch Jesu Leben, Tod und Auferstehung hindurch entfaltet. Jesus erscheint von daher als der absolute Heilsbringer. Er ist der perfekte Kommunikator des universalen Heilswillens Gottes für alle Menschen. Und er kündigt diesen Willen nicht nur an, sondern vollzieht und erfüllt ihn zugleich. Insofern ist Jesus Christus als Selbstmitteilung Gottes in einem die Vollendung menschlicher Kommunikation, die sich in ihm auf Gott hin transzendiert. Da Liebe die tiefste Weise der Kommunikation ist, richtet sich die Nächstenliebe letztendlich auf Gott, in dem sie ebenfalls wurzelt. Rahners transzendental-anthropologischer und existential-theologischer Zugang liefert nicht nur eine Explikation Christi als Gottes definitive und unüberbietbare Selbstmitteilung; gleichzeitig verlangt er nach einer existentiellen Christologie, in der das Christusgeschehen im Eintreten in den Vollzug der eigenen Existenz angeeignet wird. Diese existentielle Aneignung wird in der persönlichen Beziehung zu und Begegnung mit Jesus Christus realisiert.

Die Frage, wie Christus in unsere heutigen Kontexte zu kommunizieren sei, umfaßt also für Rahner vier verschiedene Aspekte: erstens eine Erhellung des anthropologischen Rahmens solcher Kommunikation (transzendentale Christologie), zweitens eine Entfaltung des menschlichen Suchens nach Heil und Absolutheit (suchende Christologie), drittens eine Explikation der christologischen Lehren von der Inkarnation und Auferstehung als Gottes Selbstmitteilung (orthodox kirchliche Christologie) und viertens eine pastorale Hinführung und Anregung zu einer personalen Aneignung des Christusgeschehens (existentielle Christologie).

Edward Schillebeeckx ist der Exponent hermeneutischer Christologie. Sein Ausgangspunkt und Rahmen ist die Hermeneutik. Er hat seine Konzeption 
in seinen beiden Hauptwerken "Jesus. Die Geschichte von einem Lebenden"s und "Christus und die Christen"6 ausgearbeitet. Ihm zufolge geht es der Christologie im wesentlichen um das Verständnis und die Interpretation des JesusGeschehens mit Hilfe einer Hermeneutik des ganzen Lebens Jesu. Diese beinhaltet eine Interpretation der Botschaft und Praxis Jesu, seines Leidens und Todes sowie seiner Auferstehung. Seine Geschichte soll als Gottes Geschichte mit den Menschen gelesen werden. Schillebeeckx entwickelt seine hermeneutische Christologie im Gespräch mit wichtigen Positionen der Wissenschaftstheorie, der allgemeinen Hermeneutik, der Sprachphilosophie sowie im Austausch mit der Bibelwissenschaft. Aus ersterer übernimmt er die Einsicht, daß die Wirklichkeit immer sprachlich „konstruiert" ist, daß also Sprache das entscheidende Medium der Erfahrung und der Interpretation von etwas als etwas darstellt. Die Aufgabe der Christologie ist es demnach, das in der Sprache der biblischen Texte erfahrene und interpretierte Jesus-Ereignis zu verstehen und mit unseren gegenwärtigen Erfahrungen in unseren bestimmten Kontexten zusammenzubringen. Schillebeeckx erkennt, daß das Jesus-Ereignis von Beginn der neutestamentlichen Tradition an in den Modellen und Bildern interpretiert worden ist, die damals zur Verfügung standen und die von den christlichen Gemeinden sowohl übernommen als auch neu interpretiert wurden. Ihm zufolge ist das Kriterium einer solchen Interpretation, ob diese der Botschaft und Lebenspraxis des historischen Jesus entspricht, also seiner Ankündigung der Wirklichkeit und Herrschaft Gottes, seiner befreienden und rettenden Praxis der Basileia, seiner Kommunikation der Solidarität Gottes mit den Armen, seiner Lebensgemeinschaft mit seinen Anhängern, des Festhaltens an seiner heilsamen Praxis durch all die Konflikte hindurch, in die er geriet, und die Ablehnung, die er erfuhr, bis hin zu seinem Tod am Kreuz. Laut Schillebeeckx kann Jesu Auferstehung als Gottes rettendes Handeln an ihm an der Erfahrung derer erkannt werden, die ihm nachgefolgt sind. In einer von Jesus selbst initiierten Bekehrung bekehren sie sich zu ihm als dem Christus und finden in ihm ihr endgültiges Heil. Diese Erfahrung läßt sie zu ihrer Glaubenssprache gelangen.

Christus wird Schillebeeckx zufolge durch Interpretation und Korrelation in unsere gegenwärtigen Kontexte kommuniziert. Dies impliziert erstens eine Interpretation der biblischen Termini und Texte als Ausdruck von Erfahrungen, die Menschen mit Jesus gemacht haben. Zweitens schließt die Kommunikation Christi eine Korrelation sowohl seiner Botschaft und Praxis als auch der Erfahrungen der ihm Nachfolgenden mit unseren eigenen Erfahrungen ein. Drittens besteht sie in einem Verstehen des Jesus-Ereignisses in unseren eigenen Erfahrungen, unserer Sprache und unseren Lebenskontexten. Sie zielt damit auf eine kreative Aneignung seiner befreienden und rettenden Botschaft und Praxis und darauf, ihn als Gottes Gleichnis und Paradigma des Menschen zu verstehen und zu erfahren, der nach unserer eigenen Umkehr und Praxis der Versöhnung und Befreiung ruft.

Hans Urs von Balthasar nähert sich der Christologie aus der Perspektive der Ästhetik oder genauer der Dramatik. In seiner „Theodramatik“" ten Teil seiner monumentalen theologischen Trilogie, die die Bereiche der „Theo-phanie“, der „Theo-praxie“ sowie der „Theo-logie" umfaßt, entwickelt er eine Christologie, der das Prädikat dramatisch zukommt. Jesus Christus er- 
scheint darin als ein Schauspieler im göttlichen Drama, dessen Autor Gott der Vater ist und bei dem der Geist Regie führt. Das trinitarische Drama selbst wird ermöglicht durch die ewige Zeugung des Sohnes in einer Art Ur-Kenose, die den Raum für die folgenden drei Kenosen eröffnet. Sie geschehen im Geschenk der Freiheit an die Geschöpfe, im Bund mit Israel und schließlich in der Menschwerdung des Sohnes, der seine eucharistische Haltung im "pro nobis" des Kreuzes und der Auferstehung erhellt. Balthasar betrachtet die Aussendung des Sohnes als die ökonomische Form des ewigen Ausgangs vom Vater. Das Theodrama, welches sowohl ein Drama im trinitarischen Gott selbst als auch ein Drama zwischen Gott und Mensch, das heißt ein Drama zwischen zwei Freiheiten darstellt, bewegt sich auf das Kreuz zu und kulminiert in ihm. In der Passion Jesu Christi liefert Gott seinen Sohn an die sündige Welt aus. Der Sohn trägt die Sünde der Welt, die ihn in die völlige Gottesferne und sogar in die Erfahrung des Verlustes Gottes und der Hölle führt, in der letztmöglichen Annahme seiner Sendung. ${ }^{8}$ Dadurch nimmt der wirkliche Stellvertreter der Sünde der Welt, der ausgelieferte Sohn, einen Platztausch mit den Sündern vor und unterwandert so die gottlose Welt, so daß das Wunder der Transfiguration geschehen kann, in dem die sündhafte menschliche Verwerfung Gottes durch den gehorsamen Sohn sowohl überwunden als auch unterfangen wird. Er nimmt die Sünde der Welt hinweg, indem er sie in seine Beziehung zum Vater hineinnimmt, womit der Sünder in die innertrinitarische Liebesrelation hineingenommen wird. Jesu Tod am Kreuz wird zur Darstellung der größtmöglichen Lebendigkeit des dreieinen Gottes, die selbst die größtmögliche Negation, den Tod, überwindet in die Positivität der trinitarischen Liebe und des trinitarischen Lebens hinein. Balthasar zufolge ist es zuallererst die Passion Jesu, die das immanent-trinitarische Leben und die dreieinige Liebe erschließt. Die Passion ist die „akme“ im Theodrama, die Vollendung der Sendung Jesu, der Ort seiner Stellvertretung der Sünde der Welt, des göttlichen Gerichts und der Erlösung, der Darstellung und des Vollzugs der göttlichen Liebe und die Einführung in das trinitarische Leben. ${ }^{9}$

Versucht man von diesem theodramatischen Ansatz her die Frage zu beantworten, wie Jesus Christus in unsere heutigen Kontexte zu kommunizieren sei, so kann man m. E. auf drei entscheidende Modi hinweisen. Balthasar zufolge geschieht die Kommunikation Christi zuallererst im kreuzförmigen Leben der Kirche, die seine Sendung weiterführt. Sie ist Mitarbeiterin an seiner Sendung, Mitspielerin in seinem Drama und Zeugin seiner Passion und Erlösung. ${ }^{10}$ Zweitens wird Christus in der eucharistischen Darstellung des „mysterium paschale" kommunziert, in dem Jesu Selbsthingabe vergegenwärtigt und sichtbar gemacht wird. Kommunikation Christi geschieht schließlich in der Betrachtung des göttlichen Dramas, insbesondere in der Betrachtung des österlichen Geheimnisses.

Der christologische Zugang der politischen Theologie und der Befreiungstheologie nimmt seinen Ausgang von der Perspektive befreiend-verändernder Praxis. Er bezieht sich primär auf die Praxis Jesu. Seine Praxis wird aus praktischer Sicht verstanden, nämlich von dem her, was sie für heutige christliche Praxis bedeutet. Politische und Befreiungstheologien, die sich selbst als Reflexion auf christliche Praxis in der praktischen Absicht, diese weiterzubrin- 
gen, betrachten, beginnen mit der Analyse der sozialen, politischen und kulturellen Kontexte, in denen gegenwärtige Praxis und Reflexion stattfindet, um von dort aus nach dem Kontext, der Struktur und der Intention der Praxis Jesu zu fragen. Politische wie Befreiungstheologien sind vor allem daran interessiert, die Praxis Jesu auf seine eigene "Theologie“ zu beziehen, da letztere sich aus seinem Handeln erschließt. Mit dem Anspruch, daß die Nachfolge Jesu die Voraussetzung seiner Kenntnis sei, hat die Befreiungstheologie laut Jon Sobrino "die Gestalt des historischen Jesus innerhalb der Theologie rehabilitiert" ${ }^{\text {"11 }}$. Jesu prophetische Praxis der Denunzierung böser und unterdrückerischer Handlungsstrukturen und seine Ansage der Gottesherrschaft, seine messianische Praxis des Heilens und der Befreiung werden im Kontext der politischen Situation römischer Besatzung, der sozialen Situation der Ausbeutung und Marginalisierung der Mehrheit der Bevölkerung und der religiösen Situation eines gespaltenen Frühjudentums analysiert. Diese Analyse geschieht mit dem Ziel, die Struktur und den Inhalt seiner befreienden Praxis im Blick auf die Armen und Entrechteten wie seinen Ruf in die Nachfolge herauszuarbeiten. Aus der Sicht der politischen Theologie und der Befreiungstheologie war es dieses Handeln, das Jesus mit den Repräsentanten der bestehenden Ordnung in Konflikt brachte. Seine Konfrontation mit den Mächtigen, die er nicht von seinem Verständnis Gottes und der Gottesherrschaft zu überzeugen vermochte, wurde alsbald unausweichlich. Im Laufe dieser Konfrontation mit den Vertretern des herrschenden Systems wurde Jesus selbst zum Opfer. Er fand ein schmachvolles Ende in einem politischen Tod am Kreuz. Politische wie Befreiungstheologen betrachten Jesu Auferstehung in erster Linie als Gottes solidarisches Handeln an ihm, in dem Gott Jesu Person und Praxis endgültig bestätigt, seine Person rettet und seine befreiende Praxis fortsetzt, indem er sie an ihm selbst geschehen läßt.

Nach Ansicht der politischen Theologie und der Befreiungstheologie geschieht Kommunikation Christi in unsere Kontexte dort, wo die befreiend-kommunikative Struktur der Praxis Jesu in unseren politischen, sozialen und kulturellen Situationen realisiert wird. Nachfolge Jesu passiert dort, wo Menschen in Solidarität mit den Armen handeln, wo sie gegen soziale Diskriminierung, politische Unterdrückung und kulturelle Enteignung antreten, wo sie für Befreiung kämpfen und befreiend handeln. Die politische Theologie wie die Befreiungstheologie weiß sehr wohl, daß solches Handeln in Konfrontation und Konflikte führt. Es zieht Leiden nach sich und es wird in der Praxis des Leidensgetan. ${ }^{12}$ Kommunikation Christi geschieht in Christopraxis. Diese beinhaltet die Übernahme seiner befreienden Botschaft von der Gottesherrschaft, die Übernahme seiner prophetischen Praxis, das Ertragen von Konflikten und Leiden um Christi willen, und sie zielt darauf, alle Strukturen von Herrschaft, Ausbeutung und Sünde in der Erfahrung und Praxis der Basileia zu durchbrechen.

\section{Dimensionen der Kommunikation}

Bei dem Versuch, einige Grunddimensionen der Kommunikation zu benennen, muß zuallererst die anthropologische Basis herausgestellt werden. Auf der elementarsten Ebene besagt dies, daß der Mensch ein ,animal linguale" ist, das sich der Wirklichkeit mittels Sprache und Kommunikation nähert. Seine 
Wirklichkeit ist nicht nur gesellschaftlich, sondern zugleich sprachlich konstituiert. Unsere Wirklichkeit existiert als solche nicht unabhängig von Kommunikation, so daß es keinen sprachunabhängigen Zugang zu ihr gibt. Sie ist vielmehr immer schon sprachlich konstituiert, erschlossen und ausgelegt, so daß sie sprachlich vermittelt erfahren, erkannt und verändert wird. Die Sprache ist konstitutiv für die menschliche Existenz, welche sich in Kommunikationsgemeinschaften vollzieht. Auf dieser anthropologischen Basis möchte ich einige der Einsichten skizzieren, zu denen pragmatisches Denken im Blick auf die Struktur der Sprache, des Handelns bzw. der Kommunikation gelangt ist. Die Wirklichkeit wird erfahren, erkannt und verändert, indem von allen möglichen Arten verbaler und audio-visueller Kommunikation Gebrauch gemacht wird. Aus solch pragmatischer Sicht gibt es fünf entscheidende Ebenen bzw. Dimensionen der Kommunikation, die miteinander verbunden sind und gemeinsam ein Kommunikationsmodell darstellen.

Erstens eignet der Sprache ein konstitutiv intersubjektiver Charakter. ${ }^{13}$ Sprache ist etwas dem Individuum Vorgegebenes, die es immer schon mit anderen teilt. Bei deren Erwerb in Gestalt einer bestimmten Sprache wird es zugleich in eine Kommunikationsgemeinschaft integriert, in der grundlegende Annahmen über und Zugänge zur Wirklichkeit geteilt werden. Diese sind vom Individuum in seiner Sozialisation zu übernehmen in der Weise, daß sich der Einzelne zugleich mit allen anderen identifizieren und sich von ihnen abzugrenzen lernt. Dadurch gewinnt er eine Identität und lernt, sich als Subjekt zu begreifen, somit „Ich“ zu sagen. ${ }^{14}$

Eine zweite fundamentale Einsicht pragmatischen Denkens betrifft den propositional-performativen Charakter von Sprache, die zum einen von etwas handelt und mit der zum anderen gehandelt wird ${ }^{15} \mathrm{Er}$ kommt in den elementaren Einheiten menschlicher Kommunikation, der Verwendung von Sätzen in Äußerungen zum Ausdruck. Die Äußerung ist durch eine Doppelstruktur gekennzeichnet; in ihr verbindet sich ein propositionaler Bestandteil, d.h. das, worüber die Rede ist, der in einem propositionalen Akt der Referenz und der Prädikation vollzogen wird, mit einem illokutiven Bestandteil. Dieser zeigt an, was getan wird, indem jemand etwas sagt. Der illokutive Bestandteil, mit dem der illokutive Akt vollzogen wird, legt somit fest, in welchem Sinne der propositionale Bestandteil verstanden werden soll. Eine dritte Komponente des Sprechaktes betrifft die faktischen Auswirkungen, die das Gesagte und damit Getane auf die Hörer hat. Kommen solche Wirkungen zustande, so geschieht ein perlokutiver Akt. Sprache ist demnach adäquat nur unter Einschluß der Pragmatik zu analysieren als intersubjektiver Vollzug von Sprachhandlungen, die aus Äußerungs-, propositionalen, illokutiven und intentional perlokutiven Akten bestehen. ${ }^{16}$

Mit Sprachhandlungen werden allerlei Dinge getan, nämlich soziale Beziehungen hergestellt, aufrechterhalten und verändert. Mit ihnen wird in Behauptungs-, Frage-, Bitt-, Versprechenshandlungen etc. in Wirklichkeit eingegriffen. Mit ihnen wird die innere, die soziale wie die äußere "Welt" als solche konstituiert, erschlossen, in Frage gestellt, bestritten, verändert etc. Während der propositionale Aspekt z.B. in der theologischen Kommunikation dominant 
wird, steht der performative Aspekt in der religiösen und insbesondere in der rituellen Kommunikation im Vordergrund. ${ }^{17}$ Die propositional-performative Dimension impliziert zugleich die Fragen, was denn die Wahrheit des Gehaltes konstituiert, was die richtigen bzw. gerechten intersubjektiven Beziehungen sind und in welchem Verhältnis die Wahrhaftigkeit der Kommunikationspartner zur Wahrheit und zur Richtigkeit steht. ${ }^{18}$

Drittens ist der Sprache ein konstitutiv textualer Charakter eigen. Kommunikation vollzieht sich mittels Texten. Diese Tatsache weist auf die Textualität bzw. Medialität der Sprache hin. Texte sind Medien der Kommunikation, die in ihnen auf vielfältige Weise kodiert, artikuliert und vermittelt wird. Texte treten entweder in mündlicher, schriftlicher oder audio-visueller Form auf. Ein solches Verständnis beschränkt Texte nicht auf Schrifttexte, sondern erkennt in ihnen ein Element jedweder Kommunikation. ${ }^{19}$ In kommunikativen Prozessen werden mit Hilfe von Texten vielerlei Dinge getan. Texte werden produziert, reproduziert, gebraucht und rezipiert. In Interaktion eingebettet, mit nichtsprachlichen Handlungen verwoben, haben sie bestimmte Intentionen und erfüllen bestimmte Funktionen im Hinblick auf die Verständigung der beteiligten Kommunikations- bzw. Interaktionspartner über die thematisierte Sache.

Textual vermitteltes kommunikatives Handeln geschieht viertens immer in konkreten Situationen. Es ist in seinem jeweiligen Kontext der Sprache und des Handelns lokalisiert, der die Bedeutung und die Relevanz des betreffenden Textes mitbestimmt. Kontext meint sowohl die unmittelbare Handlungssituation als auch die weitere sozio-politische und kulturelle Umgebung, in der die Kommunikation geschieht. Der kontextuelle Charakter der Kommunikation macht es nötig, sich nicht allein auf die bestimmte Äußerung, Handlung bzw. den bestimmten Text zu beschränken, sondern deren Verwobenheit mit sowie deren Mitprägung durch die Sprachspiele, Institutionen und Kulturen, zu denen sie gehören, im Blick zu behalten. ${ }^{20}$

Fünftens hat die Kommunikation schließlich eine Reihe von Zielen. Mittels Kommunikation versuchen Menschen einander zu beeinflussen, Interaktionsverhältnisse zu etablieren, aufrechtzuerhalten oder auch zu ändern, um so ihre persönlichen und kollektiven Ziele zu realisieren. Kommunikation zielt auf die Schaffung, Erhaltung und Veränderung von Gemeinschaften. Die universalbzw. transzendentalpragmatische Reflexion hat sich bemüht herauszustellen, daß es elementare Ziele von Kommunikation gibt. Kommunikation zielt ursprünglich auf Verständigung. Verständigung ist dasjenige Charakteristikum der Kommunikation, auf das die universal- bzw. transzendentalpragmatische Reflexion auf die Bedingungen der Möglichkeit sprachlicher Kommunikation bzw. auf die Grundlagen sprachlicher Verständigung abhebt. Verständigung wird als für denOriginalmodus kommunikativen Handelns bestimmend sowie als Voraussetzung kommunikativen Handelns aufgewiesen. ${ }^{21}$

Damit sind die wichtigsten Dimensionen der Kommunikation genannt. Kommunikation erweist sich als fünfdimensional. Sie ist intersubjektiv, propositional-performativ, textual, kontextuell und intentional, wobei ihre Grundintention die Ausrichtung auf Verständigung zu sein scheint. 


\section{Leitlinien für die Kommunikation Christi}

Um Christus in unsere heutigen Kontexte zu kommunizieren, bedarf es der Erfüllung bestimmter Voraussetzungen. Sie sollen im folgenden im Anschluß an unsere Skizzierung der Dimensionen der Kommunikation und im Hinblick auf die verschiedenen christologischen Zugänge erörtert werden. Um Christus zu kommunizieren, bedarf es der Berücksichtigung von fünf Dimensionen, die zugleich Leitlinien für sie darstellen. Die Kommunikation Christi muß sich ihres kontextuellen, ihres intersubjektiven, ihres objektiven, ihres intentionalen sowie ihres textualen bzw. mediativen Charakters bewußt sein.

Kommunikation Christi geschieht immer in einem bestimmten Kontext und muß von daher in diesen Kontext passen. Sie muß sich der Situation und Umgebung anpassen, in der sie stattfindet, um diese Situation überhaupt erreichen und beeinflussen zu können. Kommunikation Christi hat von daher kontextuell zu sein. Um aber kontextuell zu sein, bedarf es sowohl eines hermeneutischen Verständnisses wie einer kritischen Analyse der gegebenen Situation. Einerseits beinhaltet dies ein Verständnis der Situation der potentiellen Adressaten, ihres Vorverständnisses, ihrer Erfahrungen, ihrer Sprache, ihres kulturellen Hintergrundes, ihrer Lebensformen und Sprachspiele. Eben darauf hebt Schillebeeckx' Ansatz ab. Andererseits ist eine gesellschaftskritische Analyse ihrer politischen, sozialen und kulturellen Position, ihrer sozialen Schichtung, ihres politischen Status und ihrer kulturellen Systeme der Produktion von Sinn vonnöten. Damit beschäftigen sich insbesondere die politischen und die Befreiungstheologien. Eine konkrete kontextuelle Analyse ${ }^{22}$ wird darum zur Vorbedingung für die Kommunikation Christi, die sich sowohl in Korrelation als auch in Konfrontation mit den Erfahrungen, Fragen und Sorgen ihrer Rezipienten vollzieht. Von daher ist eine dialektische Beziehung zum Kontext notwendig. Genau dies kommt m. E. in der Pastoralkonstitution über die Kirche in der Welt von heute zum Ausdruck, wenn sie verweist auf „die Pflicht, nach den Zeichen der Zeit zu forschen und sie im Licht des Evangeliums zu deuten“ (LG 4). Durch dialektische Anpassung an die gegebene Situation einerseits und deren kritisches und kreatives Transzendieren andererseits wird eine wirkliche Kontextualisierung, Inkulturation und „Inkarnation“ der Botschaft, der Praxis und der Person Jesu Christi ermöglicht. ${ }^{23}$

Für die Kommunikation Christi ist es nicht nur notwendig, die Rezipienten in ihrem Kontext zu erreichen; zugleich muß sie ihnen Christus auf eine Weise erschließen, die sie existentiell berührt. Insofern muß es der Kommunikation Christi darum gehen, ihre Rezipienten in Adressaten zu verwandeln, in "Hörer des Wortes“, die so befähigt werden, ihm zu begegnen und in Beziehung zu ihm zu treten. ${ }^{24}$ Die Begegnung mit Christus steht in der Tat im Zentrum von Rahners Christologie. Begegnung heißt dabei nicht bloß, ihn im eigenen Leben zu erfahren, sondern auch, mit seinem Ruf in die Umkehr und Nachfolge konfrontiert $\mathrm{zu}$ werden. Christus begegnen hat von daher sowohl eine genuin personale oder existentielle als auch eine unverzichtbare intersubjektive, einschließlich einer öffentlichen oder politischen Dimension. Um zu ihm Zugang zu gewinnen, bedarf es authentischer und kompetenter Boten, die ihn in ihrer eigenen Person und Praxis sichtbar und zugänglich machen. Genau das ist es, was Zeugen sind und was sie tun. Ein Zeuge ist jemand, der in seiner Praxis und 
durch seine Person Zeugnis ablegt für das, was er gesehen und eingesehen hat, was ihm evident geworden ist. Ein Zeuge Christi zeigt mittels seiner eigenen Existenz und seines Handelns auf ihn, um ihn seinen Adressaten zugänglich, wahrnehmbar und evident zu machen. ${ }^{25}$

Die Kommunikation Christi hat sich darauf zu orientieren, ihn selbst in den Kommunikationsprozeß einzubringen bzw. diesen für seine Gegenwart transparent werden zu lassen, damit er erkannt und ihm begegnet werden kann. Dies geschieht sowohl in der eucharistischen Darstellung seines Dramas, in der Praxis seiner Jünger wie im Leiden derer, in denen die Agonie Christi bis ans Ende der Welt fortdauert. ${ }^{26}$

Die Kommunikation Christi impliziert notwendigerweise eine objektive Dimension. Sie handelt von etwas, das heißt, sie hat einen propositionalen Gehalt. Dieser propositionale Gehalt ist höchst relevant für Schillebeeckx' hermeneutische wie für politische und Befreiungschristologien, bei denen die Basileia im Zentrum steht. Der propositionale Gehalt umfaßt Jesu kommunikativbefreiende Praxis wie die in seiner Theologie im Blick auf die Gottesherrschaft und seine eigene Person und Mission erhobenen Geltungsansprüche. Sie umfaßt auch das Drama des Lebens Jesu, seiner Konfrontation mit den Mächtigen und seines Zum-Opfer-Werdens am Kreuz. Natürlich darf der propositionale Gehalt nicht auf Jesu irdisches Lebens beschränkt werden. Er hat sich auf die Wahrheitsansprüche zu beziehen, die Jesus selbst erhoben hat und die durch sein schmachvolles Ende grundsätzlich in Frage gestellt sind, und er hat sich auch auf Gottes Bestätigung dieser Ansprüche der Person Jesu zu beziehen, die in Gottes rettendem Handeln der Auferweckung erfolgt. Darum schließt der Gehalt der Kommunikation Christi den Gott Jesu Christi ein, wie er ebenso den Geist einbezieht, durch den Christus bei seinen Nachfolgern gegenwärtig ist. Auf diese Weise erschließt die Kommunikation Christi, indem sie etwas über Jesu Person, Handeln und Drama vermittelt, seine Mission im dramatischen Geheimnis Gottes. ${ }^{27}$ Sie behauptet darin, daß Gott in ihm, durch ihn und an ihm gehandelt hat und weiterhin handelt, daß Jesus nicht nur die Wahrheit sagt, wahrhaftig ist und die Wahrheit tut, sondern daß er die Wahrheit ist und daß seine Person durch den Geist gegenwärtig und wirksam ist. ${ }^{28}$ Sie behauptet damit, daß der Gegenstand ihrer Kommunikation zugleich darin als ihr Subjekt und Handlungsträger präsent ist. Gilt es auch, diese Doppelstruktur der Kommunikation im Blick zu behalten, so hat die Kommunikation Christi gleichwohl eine objektive oder auch informative Dimension. Sie informiert über den irdischen Jesus wie über den himmlischen Christus. Sie hat seine Botschaft und Wahrheit bekannt zu machen. Freilich muß dabei bedacht werden, daß dies keine neutrale, uninteressierte Information darstellt, sondern einen Versuch, sein Geheimnis und seine Mission zu erfassen. Darum geht sie zum einen immer einher mit christologischer Explikation, Interpretation und Kontemplation; andererseits bezieht sie die Kommunikatoren in einer Weise ein, daß diese nicht neutral bleiben können. Sie werden nur dann zu wahren Kommunikatoren, wenn sie sich selbst von der Wahrheit des von ihnen kommunizierten Gehaltes betreffen und bekehren lassen, wenn sie sich damit auf eine Weise der Kommunikation einlassen, die sie selbst involviert, und das heißt, wenn sie Christus bezeugen. 
Die Kommunikation Christi dient immer einem Ziel. Sie geschieht in bestimmter Absicht, verfolgt einen bestimmten Zweck, sie hat einen intendierten perlokutiven Effekt. Die Intention derer, die Christus kommunizieren, hat sich in den Dienst seiner eigenen Intentionen zu stellen, welche im Verlauf seines Lebens und seiner Praxis deutlich geworden sind. Die Intention der Mission Jesu läßt sich aus der Perspektive der politisch- und befreiungstheologischen Christologien folgendermaßen zusammenfassen: Es ging ihm darum, Menschen in der Gottesherrschaft, die in ihm nahegekommen und angebrochen ist, zu sammeln, jede Art von Trennung, Exkommunikation und Herrschaft zu überwinden, die Erfahrung seines befreienden Gottes zu teilen, den er selbst in seinem kommunikativen Handeln präsent gemacht hat, in die Praxis der Basileia einzuladen und zur Umkehr sowie zur Nachfolge zu rufen. Entsprechend Jesu eigener Kommunikation muß sich die Kommunikation Christi darauf ausrichten, Menschen im Volk Gottes zu sammeln, politische, soziale und kulturelle Exkommunikation zu überwinden. Sie muß darauf aus sein, die Erfahrung des befreienden Handelns Gotttes in ihm und an ihm mitzuteilen und mit anderen zu teilen, so daß Menschen überzeugt werden, umkehren und in seine Nachfolge eintreten. Was "Communio et Progressio" den modernen Kommunikationsmitteln zuspricht, gilt zuallererst für die Kommunikation Christi, nämlich daß durch sie „die Menschen einander noch besser kennenlernen und leichter zueinander finden" (CP 12). Die Kommunikation Christi muß das Ziel haben, zu einer wechselseitigen Verständigung mit den Adressaten zu gelangen, um sie von derjenigen Wahrheit zu überzeugen, die Jesus getan hat und die er in Person ist. Ihre Intention kann von daher einladend genannt werden, insofern sie dazu einlädt, die Erfahrung des befreienden Gottes zu teilen.

Schließlich müssen die Mittel, durch die Christus kommuniziert wird, deren kontextuelle, intersubjektive, objektive und intentionale Dimension reflektieren. Botschaft, Boten und Medien sind miteinander verknüpft. Kommunikation Christi, die auf wechselseitige Verständigung zielt und darauf, Menschen von seiner Person und Praxis zu überzeugen, muß auf kommunikative Weise vermittelt werden. Dies impliziert, daß jede Art von Manipulation, Indoktrination oder Repression zurückzuweisen ist. Sie muß sich auf eine Weise präsentieren, die die Entfaltung ihres kommunikativen Potentials des Teilens und Einladens erlaubt. Besonders relevant sind in diesem Zusammenhang jene Medien, die kontextualisiert sind, die die Vermittler einbeziehen und die zur Partizipation von seiten der Adressaten einladen bzw., besser noch, die die Adressaten zur Partizipation befähigen und zu Partizipierenden machen. Solche Medien können z. B. das liturgische Drama sein, das Menschen in sich hineinzieht und sie zu Teilnehmern am österlichen Geheimnis macht; dazu zählen verschiedene Arten von Texten, Bildern und Handlungen, und vor allem solche Mediatoren, die die Botschaft in ihrer eigenen Person verkörpern. Nur authentische und kompetente Boten sind in der Lage, den Inhalt ihrer Kommunikation überzeugend zu vermitteln. Nur sie können selbst zu Medien werden, die den kommunizierten Christus in ihrer eigenen Praxis und Person zugänglich, sichtbar und verständlich machen und die damit für Christus transparent werden. Genau das ist die Rolle und Aufgabe des Zeugen, durch den die mediative Dimension der Kommunikation Christi am deutlichsten zum Tragen kommt. 


\section{Kommunikation Christi und christliche Gemeinschaft}

Die Kommunikation Christi geht vonstatten in einer Gemeinschaft, die eine bestimmte Kommunikationsgemeinschaft darstellt. Sie geschieht in der und durch die Kommunikationsgemeinschaft namens Kirche. Innerhalb der Kirche wird Jesus durch eine Viclzahl von Handelnden und Handlungen kommuniziert. Aus meiner Sicht können diese sowohl systematisch als auch praktisch im Rahmen einer theologischen Handlungstheorie reflektiert werden. Dieser Ansatz wurde von Helmut Peukert im Gespräch mit der Wissenschaftstheorie dieses Jahrhunderts, mit Habermas' Theorie des kommunikativen Handelns und mit vorliegenden theologischen Konzeptionen entwickelt. In seinem Buch „Wissenschaftstheorie - Handlungstheorie - Fundamentale Theologie ${ }^{\text {“29 }}$ entfaltet Peukert den Ansatz und Status politisch-theologischer Theoriebildung als einer Reflexion auf Praxis. Er gelangt damit zu einer "theologischen Theorie kommunikativen Handelns ${ }^{\text {“30. }}$.

Im Durchgang durch die wissenschafts- und handlungstheoretische Diskussion zeigt er auf, daß diese mehr und mehr auf das kommunikative Handeln als die Basis auch wissenschaftlicher Rationalität stößt. Er stellt heraus, daß die Anliegen der Wissenschafts- und Handlungstheorien in einer Theorie des kommunikativen Handelns, wie sie Habermas entwickelt hat, konvergieren. Zugleich macht Peukert deutlich, daß es innerhalb von Habermas' Theorie eine fundamentale Aporie gibt. Diese Aporie zeigt sich darin, daß er Egalität, Reziprozität und Solidarität als normativen Kern von Kommunikation und Interaktion aufweist, ohne in der Lage zu sein, diese als unbegrenzt und damit wirklich universal zu denken. Peukert behauptet dann, „daß es in der jüdischchristlichen Tradition um die Wirklichkeit geht, die in den Grund- und Grenzerfahrungen kommunikativen Handelns erfahren wird, und um die Weise kommunikativen Handelns, die angesichts dieser Erfahrungen noch möglich ist". Und er behauptet ferner, "daß eine fundamentale Theologie als Theorie dieses kommunikativen, anamnetisch-solidarisch auf den Tod zugehenden Handelns und der in ihm erfahrenen und erschlossenen Wirklichkeit entwickelt werden kann und muß ${ }^{\text {"31 }}$.

Peukerts Ansatz kann als eine kritische Theorie christlich-kommunikativer Praxis gelten. Er ist durch und durch interdisziplinär orientiert, legt die Grundlagen politischer Theologie und zielt auf eine politisch-theologische Theorie, die eine kritische Theorie des Subjekts, der Gesellschaft und der Geschichte umfaßt. Ich habe versucht, Peukerts Ansatz einer kommunikativen politischen Theologie in biblischer wie in systematisch-theologischer Perspektive weiterzuführen, indem ich in meiner pragmatischen Gleichnistheorie ${ }^{32}$ Jesu kommunikative Praxis untersucht und indem ich mich bemüht habe, die Grundstruktur christlicher Glaubenspraxis zu enieren in dem, was ich elementare Handlungen des Glaubens nenne. Ich betrachte die Handlungen des Bezeugens und Bekennens als solche Grundformen christlicher Praxis, und ich habe ihre Relevanz und ihre kritische wie politische Bedeutung aus biblischer, kirchenund dogmengeschichtlicher sowie fundamentaltheologischer Sicht zu zeigen versucht. $^{33}$ 
Christen kommunizieren Jesus Christus durch Bezeugen, darin machen sie ihn zugänglich und bringen öffentlich zum Ausdruck, was er in seinem Handeln und was Gott in und an ihm getan hat. Christen sind Zeugen, die auf die apostolischen Urzeugen und auf das Zeugnis der Jünger Jesu angewiesen sind. Jenen Urzeugen ging es darum, anderen mitzuteilen, was sie mit und in Jesus erfahren haben. Sie wollten die Wirklichkeit des auferstandenen Christus kommunizieren, wollten Jesu Person und Praxis bekannt machen, um so andere Menschen für ihn zu gewinnen, sie einzuladen in seine Nachfolge. Sie handelten darin in Übereinstimmung mit Jesu Auftrag: „Ihr sollt meine Zeugen sein!“ (Lk 24,28), um Menschen von Jesus zu überzeugen, auf daß sie sich bekehrten und ihn bekannten. Ihr Bezeugen wurde vom Zeugnis des Geistes geleitet und es zielte darauf, Menschen zu gewinnen. Insofern hatte ihre Praxis eine missionarische Intention. Diese Intention wird von Christen in dem weitergeführt, was ich kerygmatisch-missionarisches Bezeugen nenne. ${ }^{34}$ Es wird von Menschen getan, die Zeugen „zweiter Hand“35 sind. Als Zeugen zweiter Hand sind sie auf die biblischen Zeugen erster Hand angewiesen. Wie diese biblischen Zeugen kommunizieren auch sie Christus, machen Jesu Person und Praxis den Menschen bekannt, auf daß sie in eine Beziehung zu ihm eintreten und sich von ihm bestimmen lassen. Kerygmatisch-missionarisches Bezeugen zielt darauf, Menschen von der Person Jesu, von seiner Praxis der Gottesherrschaft zu überzeugen, sie in die Nachfolge seiner Person und Praxis einzuladen.

Neben dem kerygmatisch-missionarischen gibt es noch andere Formen des Bezeugens. Eine ist das diakonische Bezeugen. ${ }^{36}$ Diese Handlung des Glaubens richtet sich ebenfalls an andere, aber sie intendiert nicht primär, sie zu überzeugen, sondern zu trösten, Menschen beizustehen und Hilfe zu leisten in ihrem Leiden. Diakonisches Bezeugen ist eine solidarische Praxis, die darauf aus ist, das Leid, das Menschen zu ertragen haben, zu mildern, sie von ihren Bedrängnissen und allen Formen von Unrecht, das ihnen angetan wird, zu befreien. Diakonisches Bezeugen geschieht sowohl durch karitatives wie durch politisches Handeln. In dieser Praxis der Solidarität wird Jesus kommuniziert, ob sie sich nun explizit auf ihn bezieht oder nicht. Insofern zielt auch das diakonische Bezeugen darauf, jene universale Solidarität zu demonstrieren, die sich in Jesu Person und Praxis erschließt.

Eine dritte Form ist das prophetische Bezeugen. Es ist insbesondere für die politisch-theologische und die befreiungstheologische Christologie von Bedeutung. ${ }^{37} \mathrm{Im}$ Johannesevangelium bezieht sich das Bezeugen auf das Gericht, es kritisiert bzw. fällt Urteile, und genau das macht prophetisches Bezeugen aus. Prophetisches Bezeugen denunziert die herrschenden Verhältnisse, es deckt deren Unrecht auf und versucht, die dafür Verantwortlichen zu bekehren. Der prophetische Zeuge zielt auf Solidarität mit den Opfern von Unterdrückung, Unrecht und Ausbeutung. Durch seine Anklage versucht er, die Ausbeuter und Unterdrücker von ihrem ungerechten Tun abzubringen. Prophetisches Bezeugen intendiert ineins mit seiner Solidarität mit den Opfern die Veränderung der Unterdrückungsstrukturen, und es beabsichtigt, derer Unterstützer dazu zu gewinnen, eine gerechte und menschliche Ordnung mit aufzubauen, eine Ordnung, die dem Willen Gottes entspricht. 
Schließlich komme ich zu dem, was ich pathisches Bezeugen nenne. Es ist sowohl für Rahner, Schillebeeckx und von Balthasar als auch für politischtheologische und Befreiungschristologien relevant. ${ }^{38} \mathrm{Im}$ letzten Buch des Neuen Testaments wird der Märtyrer zum Zeugen par excellence. Im frühen Christentum wurde der Begriff "martys" alsbald zur exklusiven Bezeichnung derer, die um Christi willen hatten sterben müssen, deren Blut um ihres Glaubens willen vergossen worden war. Pathisches Bezeugen ist die äußerst mögliche Handlung des Glaubens. Wer immer um Christi willen verfolgt, gefoltert und getötet wird, macht Christus auf die höchstmögliche Weise sichtbar. Er wird ein „Zeuge des Leidens Christi“ (1 Petr 5,1); er "tritt in die Fußstapfen der Passion des Herrn“"39. Der pathische Zeuge identifiziert sich mit Jesu Person, Praxis und Passion in einem solchen Ausmaß, daß er mit ihm leidet. Ignatius von Antiochien sprach von „sympaschein “40. Pathisches Bezeugen gab es nicht nur zu Zeiten des Frühchristentums. Im Gegenteil, es ist weltweit hochaktuell. Meines Erachtens zielt es ebenfalls darauf, die Verfolger für Christus zu gewinnen. Im Angesicht seiner Verfolger macht der Zeuge ein letztes Angebot, sie zu überzeugen. Indem er zum Märtyrer wird, demaskiert er deren Handeln als mörderisch; er deckt damit zugleich deren Unwahrheit auf.

Bezeugen ist also eine elementare Handlung des Glaubens, mit der Christen in ihrem eigenen Handeln in verschiedenen Situationen und Kontexten auf Christus hinweisen, seine Person und Praxis zugänglich machen und ihn in ihrem kerygmatisch-missionarischen, diakonischen, prophetischen und pathischen Bezeugen ihren Zeitgenossen erschließen und mitteilen.

Eine weitere elementare Handlung des Glaubens ist meiner Ansicht nach das Bekennen. Ich sehe den Hauptunterschied zwischen Bezeugen und Bekennen darin, daß letzteres nicht darauf zielt, Menschen zu überzeugen, sondern in der Tat eine gemeinsame Überzeugung zum Ausdruck bringt. Während Bezeugen von Einzelnen, Gruppen, Gemeinden und der Kirche getan werden kann, bezieht sich das Bekennen immer auf die Kirche selbst. Insofern stellt es eine ekklesiale Handlung dar. Deren primäre Handlungsträgerin ist die Kirche, die im Akt des Bekennens einen Konsens, d. h. ein Bekenntnis im Sinne einer Selbstverständigung formuliert, die zugleich eine Grenzziehung beinhaltet. Auch das Bekennen bezieht sich primär auf Jesus Christus; es steckt den Rahmen für die weitere Kommunikation Christi ab. Ich unterschiede wiederum drei Formen des Bekennens. Bekennen hat seinen primären Ort im Gottesdienst. Als solches ist es liturgisches Bekennen; im Rahmen der Feier des eucharistischen Dramas ist es Teil ritueller Kommunikation..$^{41}$ Die ersten christlichen Gemeinden bekannten bereits Christus als ihren Herrn, akklamierten ihm während ihrer Gottesdienste. Solch liturgisches Bekennen wurde bald zum konstitutiven Element der christlichen Taufe. ${ }^{42}$ Das Taufbekenntnis ist für die Kirche grundlegend geworden. Liturgisches Bekennen geschieht auch in Verbindung mit der Feier der Eucharistie. Darin hat es seinen Ort im Beten des Credos als Antwort auf das Evangelium. Es wird zur Doxologie, in der die Gemeinde Gott dankt und ihren Herrn Jesus Christus lobpreist. Meines Erachtens gehört auch das Sündenbekenntnis zum liturgischen Bekennen. Es hat in erster Linie eine ekklesiale Funktion, wie von der Praxis der öffentlichen Buße in der alten Kirche gezeigt 
werden kann, wo das Sündenbekenntnis zur Versöhnung mit Gott und mit der Kirche führt.

Neben dem liturgischen Bekennen gibt es eine weitere ekklesiale Handlung des Glaubens, die ich lehrendes Bekennen nenne. Es läßt sich bis auf die antihäretischen Formeln im Neuen Testament, vor allem in den Johannesbriefen, zurückführen. Lehrendes Bekennen ist mit den dogmatischen Auseinandersetzungen des 4. und 5. Jahrhunderts in den Vordergrund getreten, und es hat zu einer Reihe von Bekenntnissen geführt, die von Synoden und Konzilien formuliert worden sind. Diese Versammlungen hatten die Absicht, einen bestimmten in der Kirche erreichten Konsens zu fixieren, und sie haben diesen Konsens in Form von Credos oder anderen Bekenntnistexten formuliert, von denen einige später ebenfalls liturgisch gebraucht wurden.

Im 20. Jahrhundert ist eine neue Art von Bekenntnis und eine neue Form des Bekennens entstanden. Ich nenne diese situatives Bekennen. ${ }^{43}$ Es ist dies ein Bekennen im Kontext des Kampfes, in dem deutlich Position bezogen und damit Partei ergriffen wird. Es ist gekennzeichnet von einem neuen Verständnis der Verbundenheit von Wahrheit und Praxis, Situation und Lehre. Solches Bekennen geschieht, wo die Kirche in einer bestimmten geschichtlich-politischen Situation gezwungen ist zu bekennen, wo sie "ja“ oder "nein" zu sagen hat, wo von ihr um ihrer eigenen Identität, Integrität und Mission willen ein entscheidender Konsens gefordert ist.

Wo Bezeugen zum Konsens führt, geschieht Bekennen. Das Bekennen Christi ist der Vollzug und die Darstellung des ekklesialen Konsens. Während es beim Bezeugen im wesentlichen um eine kreative, kontextuelle Aneignung der Kommunikation Christi geht, bezieht sich das Bekennen anamnetisch auf die Grundlage dieser Kommunikation, und es bringt eben diese verbindliche Basis vor Gott, voreinander und vor der Öffentlichkeit zur Sprache. Im Bekennen erinnert die Kirche sowohl an den verbindlichen Grund ihrer Existenz wie an deren Ziel, teilt beide mit, indem sie sich „vor den Menschen“ (Lk 12,8) zu Christus bekennt.

„Communio et Progressio“ nennt Christus „communicator perfectus“ (CP 11). Angesichts der Tatsache, daß die Standards der Perfektion im nordatlantischen Kontext von der Kulturindustrie gesetzt werden, halte ich diese Formulierung für irreführend. In Übereinstimmung mit dem Johannesevangelium ziehe ich es darum vor, Christus den wahren Kommunikator zu nennen. Er sagt die Wahrheit, ist wahrhaftig, er tut die Wahrheit, und er ist die Wahrheit in Person.

\section{Anmerkungen:}

1 Deutsche Fassung eines Textes, der beim 6. Internationalen Cavalletti Seminar vorgetragen wurde, das unter dem Thema „The Foundations for a Theology of Communication. Can Christ be said to be the 'Perfect Communicator'?" vom 16.-23. September 1991 in der Villa Cavalletti, Grottaferrata, stattfand.

2 Vgl. besonders seine entsprechenden Artikel in IThK und SM.

3 Freiburg 1976. 
4 Vgl. K. Rahner, Grundkurs $288 \mathrm{f}$

5 Freiburg 1974.

6 Freiburg 1978; vgl, auch sein methodologisch wichtiges Buch: Glaubensinterpretation, Mainz 1971.

74 Bde., Einsiedeln 1973-1983.

8 Vgl. H.U. von Balthasar, Theologie der drei Tage, Einsiedeln ${ }^{2} 1990$.

9 Balthasars theodramatischer Ansatz wird insbesondere im Blick auf das Drama des Lebens Jesu aufgenommen und weitergeführt bei R. Schwager. Vgl. seine Diskussion von Balthasars in R. Schwager, Der wunderbare Tausch, München 1986, 273-312; ders., Jesus im Heilsdrama, Innsbruck/Wien 1990.

$10 \mathrm{Im}$ Blick auf die ekklesialen Konsequenzen von Balthasars Ansatz vgl. M. Kehl, Kirche als Institution, Frankfurt 21978, 239-311.

$11 \mathrm{~J}$. Sobrino, Christology at the Crossroads, London ${ }^{3} 1984$, 79; von den vielfältigen Beiträgen der Befreiungstheologie zur Christologie vgl. etwa L. Boff, Jesus Christus der Befreier, Freiburg 1986; H. Echegaray, The Practice of Jesus, Maryknoll (NY) 1984; A. Nolan, Jesus before Christianity, Maryknoll (NY) 61984; J. M. Bonino (ed.), Faces of Jesus, Maryknoll (NY) 1984. Jesu kommunikative Praxis kommt aus politisch-theologischer Perspektive in den Blick bei H. Peukert, Wissenschaftstheorie - Handlungstheorie - Fundamentale Theologie, Frankfurt ${ }^{2}$ 1988; vgl. E. Arens, Kommunikative Handlungen, Düsseldorf 1982; J. Moltmann, Der Weg Jesu Christi, München 1989; vgl. die Reflexion auf die praktische Struktur des christlichen Glaubens bei J. B. Metz, Glaube in Geschichte und Gesellschaft, Mainz ${ }^{5} 1991$; M. L. Lamb, Solidarity with Victims, New York 1982.

12 Vgl. R. Chopp, The Praxis of Suffering, Maryknoll (NY) 1986; sowie das CONCILIUMHeft „Martyrium heute“, Conc (D) 19 (1983) H. 3.

13 Intersubjektiv in diesem Sinne bedeutet keineswegs, daß dieser Zugang sich auf die interpersonale Ebene beschränkt. Im Gegenteil zielt er darauf, sowohl die interpersonale, die der Gruppe sowie die Ebene der Gesellschaft zu umfassen.

$14 \mathrm{Vgl}$. G. H. Mead, Geist, Identität und Gesellschaft, Frankfurt 1973; J. Habermas, Theorie des kommunikativen Handelns, Bd. 2, Frankfurt 1981.

15 Das ist die grundlegende Erkenntnis der Sprechakttheorie, wie sie von John L. Austin und John R. Searle entwickelt, von Habermas und Apel rezipiert und weitergeführt worden ist; vgl. J. L. Austin, Zur Theorie der Sprechakte, Stuttgart 1972; J. R. Searle, Sprechakte, Frankfurt 1971; K.-O. Apel (Hg.), Sprachpragmatik und Philosophie, Frankfurt 1976; J. Habermas, Vorstudien und Ergänzungen zur Theorie des kommunikativen Handelns, Frankfurt 1984

16 Gegen Habermas' einseitig negative Bewertung der Perlokution (vgl. ders., Theorie des kommunikativen Handelns, Bd. 1, 390ff.) halte ich diese, soweit sie über illokutives Verstehen vermittelt ist, auch in verständigungsorientierten Sprechhandlungen für angezielt; vgl. E. Arens, Kommunikative Handlungen $237 \mathrm{f}$.

17 Vgl. im Blick auf die rituelle Praxis bzw. Kommunikation V. Turner, Das Ritual Frankfurt/New York 1989.

18 Den Zusammenhang dieser Fragen behandelt Habermas in seiner Wahrheitstheorie; vgl. J. Habermas, Wahrheitstheorien, in: ders., Vorstudien 127-182.

19 Diese zentrale Erkenntnis der Texttheorie ist bisher von der Theorie des kommunikativen Handelns nicht rezipiert worden; vgl. dazu etwa S. J. Schmidt, Texttheorie, München 1973; ders., Grundriß der Empirischen Literaturwissenschaft, Neuausgabe, Frankfurt 1991.

20 Die Kontextualität der Sprache ist vor allem von Wittgenstein und den von ihm beeinflußten Theoretikern herausgearbeitet worden; vgl. L. Wittgenstein, Philosophische Untersuchungen, Franfurt ${ }^{3} 1975$; P. Winch, Die Idee der Sozialwissenschaften, Frankfurt 1974.

21 Vgl. J. Habermas, Vorstudien; K.-O. Apel, Transformation der Philosophie, Bd. 2, Frankfurt 1976.

22 Vgl. R. J. Schreiter, Constructing Local Theologies, Maryknoll (NY) 1985.

$23 \mathrm{Vgl}$. A. Shorter, Toward a Theology of Inculturation, London 1988, bes. 59-88.

24 Vgl. K. Rahner, Grundkurs, 298-303; J. Heinrichs, „Persönliche Beziehung zu Jesus 
Christus“. Skizze zu einer handlungstheoretischen Christologie, in: ThPh 54 (1979) 50-79; H. P. Siller, Handbuch der Religionsdidaktik, Freiburg 1991, 64-67, 169-188.

25 Vgl. E. Castelli (éd.), Le témoignage, Paris 1972; darin besonders die Beiträge von E. Castelli, P. Ricoeur, X. Tilliette und K. Rahner; vgl. H. U. von Balthasar, Theodramatik Bd. II/2, 416-424; E. Arens, Bezeugen und Bekennen, Düsseldorf 1989.

26 Vgl. B. Pascal, Pensées Nr. 53.

27 Vgl. v. a. H. U. von Balthasar, Theodramatik, der aus dramatischer Perspektive die Bedeutung des Vollzugs dieses Geheimnisses in der rituellen Praxis des eucharistischen Dramas herausstreicht. Politische und Befreiungstheologien heben ab auf die politische Praxis als jenem Handlungsfeld, in dem Christopraxis geschieht.

28 Ich habe versucht, dieses johanneische Verständnis von Wahrheit zu entfalten, indem ich I. de la Potteries Einsichten (vgl. ders., La vérité dans Saint Jean, 2 vols., Rome 1977; ders., Geschichte und Wahrheit, in: R. Latourelle/G. O'Collins (Hg.), Probleme und Aspekte der Fundamentaltheologie, Innsbruck 1985, 112-135) mit Habermas' und Apels Wahrheitstheorien verbinde. Vgl. E. Arens, Zur Struktur theologischer Wahrheit. Überlegungen aus wahrheitstheoretischer,biblischer und fundamentaltheologischer Sicht, in: ZKTh 112 (1990) 1-17.

29 Frankfurt ${ }^{2} 1988$.

30 A. a. O., 351; vgl. ders., Art. Fundamentaltheologie, in: NHThG 2, 16-25; ders., Kommunikatives Handeln, Systeme der Machtsteigerung und die unvollendeten Projekte Aufklärung und Theologie, in: E. Arens (Hg.), Habermas und die Theologie, Düsseldorf 21989, 39-64.

31 H. Peukert, Wissenschafstheorie 316.

$32 \mathrm{Vgl}$. E. Arens, Kommunikative Handlungen; ders., Towards a theological theory of communicative action, in: Media Development 28 (1981) no. 4, 12-16; ders., Metaphorische Erzählungen und kommunikative Handlungen Jesu, in: BZ 32 (1988) 5271.

33 Vgl. E. Arens, Bezeugen und Bekennen; ders., Elementare Handlungen des Glaubens, in: O. Fuchs (Hg.), Theologie und Handeln, Düsseldorf 1984, 80-101; ders., Jesus' Communicative Actions: The Basis for Christian Faith Praxis, Witnessing and Confessing, in: The Conrad Grebel Review 3 (1985) 67-85; ders., Christopraxis. Grundzüge theologischer Handlungstheorie, Freiburg 1992. Eines meiner Anliegen ist zu zeigen, daß es sich beim Bezeugen und Bekennen nicht bloß um privatistischpietistische Akte handelt, sondern daß dies öffentliche oder politische Handlungen sind, die in der Bibel wurzeln, in der Geschichte der Kirche einen wichtigen Platz einnehmen, ökumenisch relevant sind und sowohl eine systematisch-theologische wie eine praktische Dimension besitzen.

34 Die Beziehung von Zeugnis und Mission steht im Zentrum der Christologien Rahners und von Balthasars.

35 S. Kierkegaard hat zwischen Schülern „erster" und „zweiter Hand“ unterschieden; vgl. im Blick auf Jesu Auferstehung K. Rahner, Grundkurs 269-272.

36 Die diakonische Dimension der christlichen Glaubenspraxis wird von Rahner in seiner "suchenden Christologie" unter dem Stichwort „Nächstenliebe" herausgestellt; sie wird unter dem Stichwort "Solidarität" von politischen und Befreiungstheologien thematisiert. Vgl. J. B. Metz, Glaube; H. Peukert, Wissenschaftstheorie; J. L. Lamb, Solidarity; G. Baum, Compassion and Solidarity, New York 1990; J. Sobrino/J. Hernández Pico, Theology of Christian Solidarity, Maryknoll (NY) 1985; vgl. auch E. Arens, Internationale, ekklesiale und universale Solidarität, in: Orientierung 53 (1989) 216-220; ders./O. John/P. Rottänder, Erinnerung, Befreiung, Solidarität. Benjamin, Marcuse, Habermas und die politische Theologie, Düsseldorf 1991.

37 Laut G. Gutiérrez geht es der Befreiungstheologie wesentlich darum, die herrschende ungerechte Ordnung zu denunzieren und die Gottesherrschaft anzusagen; vgl. sein grundlegendes Werk: Theologie der Befreiung, Mainz ${ }^{10} 1991 ;$ M. H. Ellis/O. Maduro (eds.), The Future of Liberation Theology. Essays in Honour of Gustavo Gutiérrez, Maryknoll (NY) 1989.

38 Laut Schillebeeckx, Christus, ist Leiden die wichtigste Kontrasterfahrung im Gegenüber zum Heil; von Balthasar hat verschiedentlich die Bedeutung des Martyriums 
herausgestellt (vgl. etwa Cordula oder der Ernstfall, Einsiedeln ${ }^{4}$ 1987); die politische Dimension des Martyriums ist Gegenstand verschiedener Beiträge im CONCILIUMHeft „Martyrium heute“, vgl. v. a. die Aufsätze von K. Rahner und L. Boff; vgl. J. Moltmann, Weg, 219-226; L. Kaufmann/N. Klein, Ökumene der Märtyrer, in: E. Schillebeeckx (Hg.), Mystik und Politik (FS J. B. Metz), Mainz 1988, 383-393.

39 Irenäus, Contra Haereses II 18,5.

40 Epistula ad Smyrnaeos 4,2.

$41 \mathrm{Vgl}$. H. U. von Balthasar, Theodramatik.

42 Das zeigt sich an der aus dem 2. Jahrhundert stammenden Interpolation von Apg 8,37 .

43 Ihr erstes Beispiel war die „Barmer Erklärung" der deutschen Bekennenden Kirche. Ein weiteres Beispiel ist das Bekenntnis der Würzburger Synode der Bistümer in der Bundesrepublik Deutschland von 1975 unter dem Titel „Unsere Hoffnung“. M. E. geht auch das von südafrikanischen Christen 1985 erstmals veröffentlichte KAIROSDokument, obgleich es nicht von einer offiziellen Kirchenkörperschaft verabschiedet wurde, zumindest in Richtung situativen Bekennens.

\section{SUMMARY}

The article attends to the question in what way Christ is to be communicated in our present day contexts. For this purpose the author outlines four contemporary approches to christology and examines their christologic models of communication. He then extracts a five-dimensional model of communication from current pragmatics applied in the act of developing theories, with which guidelines for the communication of Christ are being demonstrated. Finally, the relationship between the communication of Christ and the Christian community is being analysed, in which testifying and confessing are being made explicit as two different actions. Within the community of the church these serve for the formulation and conservation as well as the creative continuation of communication of Christ.

\section{RÉSUMÉ}

L'étude est consacrée à la question: comment communiquer le Christ dans les contextes d'aujourd'hui? A cet effet, elle esquisse 4 approches contemporaines de la Christologie et en èclaire les modèles chrétiens de la communication. Ensuite, elle extrait de l'actuelle théorie pragmatique un modèle de communication en 5 dimensions, à l'aide duquel de lignes de conduite seront traçées pour la communication du Christ. A la fin de l'étude, c'est la relation entre la communication et la Communauté Chrétienne qui est examinèe. Là, témoignage et profession de foi, y sont présentés telles 2 activités qui à l'intérieur de l'Eglise, servent à formuler, à garder et à prolonger de façon créative la communication du Christ.

\section{RESUMEN}

El escrito se pone la pregunta cómo se puede, en nuestro actual contexto, comunicar a Cristo. Bosqueja cuatro accesos contemporáneos a la cristología e ilustra sus modelos cristólogicos de la comunicación. Extrae de la actual pragmática formación de teoría un modelo de comunicación pentadimensional con la ayuda del que se muestran los lineamientos para la comunicación de Cristo. Finalmente se examina la relación entre la comunicación y la comunidad cristiana, en la que el arrepentimiento y el reconocimiento de profesión de fé son explicitados como dos acciones que dentro de la comunidad de comunicación la Iglesia sirven para la formulación, la mantención y la continuación creativa de la comunicación de Cristo. 